\title{
Aproximación a un modelo de innovación en la docencia universitaria
}

\author{
Max Römer PieRETti \\ Universidad Carlos III Madrid \\ mroemer@hum.uc3m.es \\ Celia CAmilli Trujillo \\ Centro Universitario Villanueva (UCM) \\ ccamilli@villanueva.edu \\ Ernesto LÓPEZ GÓMEZ \\ Centro Universitario Villanueva (UCM) \\ elopezg@villanueva.edu
}

Recibido: 22/11/2012

Aceptado: 23/01/2013

\section{Resumen}

El uso de metodologías activas es uno de los referentes del EEES. Estas suponen nuevos retos para el docente universitario, desafíos que se han asumido en la asignatura "Periodismo en la Red" que se imparte en el Grado de Periodismo de la Universidad Carlos III de Madrid. La experiencia innovadora presentada permite expresar las ventajas que ofrecen el portafolio y el aprendizaje colaborativo asistido por ordenador, con evidencias en la elaboración por parte de los estudiantes de periódicos digitales. Así, se ha elaborado un modelo de innovación de docencia universitaria, en donde ambas metodologías requieren de un compromiso tutorial del docente para el desarrollo formativo, como herramientas de trabajo que permiten a los estudiantes compartir con sus compañeros de grupo sus análisis, correcciones y evaluaciones del trabajo periodístico.

Palabras clave: aprendizaje colaborativo asistido por ordenador, tutoría universitaria, portafolio, periodismo digital, EEES.

\section{Approach to a Model of Innovation in University Teaching}

\begin{abstract}
The use of active methodologies is one of the key principles of the EHEA These suppose new challenges to the professor, challenges that have been made in the course "Network journalism" that is taught in the Degree of Journalism at the University Carlos III of Madrid. The experience in this research is the result of the implementation of these methodologies has allowed active express the advantages for teaching with tangible results in the development of digital newspapers. Thus the portfolio, mentoring and ComputerSupported Collaborative Learning (CSCL) shown have allowed work to allow students to share with their fellow group analysis, corrections and assessments of their journalistic work.

Keywords: computer-supported collaborative learning, tutoring, portfolio, digital journalism, EHEA.

\section{Referencia normalizada}

RÖMER PIERETTI, Max; CAMILLI TRUJILLO, Celia; y LÓPEZ GÓMEZ, Ernesto (2013): “Aproximación a un modelo de innovación en la docencia universitaria". Estudios sobre el mensaje periodístico. Vol. 19. Núm. especial abril, págs.: 971-979. Madrid, Servicio de Publicaciones de la Universidad Complutense.
\end{abstract}

Sumario: 1. Introducción. 2. Desarrollo: Una experiencia innovadora en la enseñanza de "Periodismo en la Red"; 2.1. El contexto innovador; 2.2. Portafolio y Aprendizaje Colaborativo Asistido por Ordenador (CSCL) como metodologías innovadoras. 3. Metodología. 4. Resultados. 5. Conclusiones. 6. Referencias bibliográficas. 


\section{Introducción}

La actualidad universitaria presenta muchos interrogantes. El sentido de la universidad, su gobierno y financiación, su relación con el sistema social, los nuevos planes de estudio, la participación estudiantil y el quehacer del profesor universitario son, entre otros, temas que están en cualquier discusión universitaria. Todos ellos nos sitúan en torno al quehacer del profesor universitario en el contexto actual, especialmente con el emergente Espacio Europeo de Educación Superior (Budapest-Vienna Declaration, 2010).

La actividad del profesor universitario se caracteriza por la diversidad, si bien, suelen identificarse dos dimensiones básicas: la docencia y la investigación, a la que últimamente se ha incorporado otra gerencial o burocrática -de gestión-. Detengámonos en la primera de ellas, la docente, con la pretensión de elaborar una caracterización de la misma fundamentada sobre tres horizontes (Ibáñez-Martín, 1990):

La docencia requiere preparación. Esta cuestión obvia admite afirmar que dicha capacitación ha de ser triple: curricular, desde un conocimiento de la discusión científica actual; actitudinal, capaz de desarrollar modos y cualidades que permitan conectar con los estudiantes; y finalmente, pedagógica, que haga posible la formulación de un programa secuencial, realista y actualizado que permita facilitar el horizonte formativo pretendido.

El discurso del profesor, eje de la docencia. La claridad y la rigurosidad son elementos clave pero más aún es la preocupación que tiene que tener el profesor por implicar al alumno en la captación del discurso, preguntándose como despertar el interés del estudiante por lo enseñado que les permita una reflexión personal. La docencia va más allá de la transmisión de conocimiento y el profesor debe poner en marcha iniciativas (seminarios voluntarios, bibliografía actualizada, acción orientadora, entre otras) para que el discurso captado por el estudiante adquiera raíces en los alumnos.

La comprobación de lo captado por el alumno, elemento fundamental de la docencia. En efecto, la dimensión evaluadora de la docencia ha de tener proyección formativa, para lo que resulta fundamental armonizar los objetivos de la materia, las competencias pretendidas, los contenidos seleccionados y los medios de evaluación empleados. Una evaluación fundamentada en la justicia, que haga posible a cada estudiante conocer las razones de la evaluación alcanzada.

Estos elementos básicos de la dimensión docente requieren una continua revisión si se pretende orientar la docencia hacía la mejora continua. La base de la mejora es un proceso permanente de reflexión-indagación, de forma sistematizada y con matiz evaluativo sobre la labor docente llevada a cabo. Por consiguiente surge el concepto de innovación.

Innovar en la docencia requiere la previa consideración de la educación como una realidad dinámica y cambiante sobre la que poder construir mejoras permanentes. Innovar no es solo hacer cosas nuevas sino, es sobre todo, ser capaces de mejorar una situación o realidad desde acciones nuevas (Medina, 2009). Por lo tanto, no siempre el cambio es garantía de innovación así como tampoco no toda innovación conduce a una mejora.

Los procesos innovadores que se relacionen con metodologías docentes necesitan de sólidos momentos de reflexión donde se detecten necesidades y claves que orien- 
ten la acción. Se requiere entonces de una investigación profunda que culmine en acciones pertinentes que permitan avanzar en vistas a ofrecer al alumnado universitario una formación de calidad.

No se trata entonces de resistirse a la innovación con la única justificación del anclaje a la tradición, ni de innovar sin una reflexión e investigación previa, más bien de encontrar evidencias fruto de la reflexión y de la investigación que sugieran la pertinencia de la innovación.

En este trabajo presentamos una experiencia de innovación docente apoyada en el uso de metodologías activas, en este caso el uso del Portafolio y del Aprendizaje Colaborativo Asistido por Ordenador (del inglés, Computer-Supported Collaborative Learning, en adelante CSCL). La experiencia se enmarca en la asignatura de "Periodismo en la Red" que se imparte en el Grado de Periodismo de la Universidad Carlos III de Madrid. Es la continuación de una línea de investigación centrada en metodologías de enseñanza-aprendizaje activas (Römer y Camilli, 2012; López, 2012), enfocada en la implementación de estrategias docentes que proporcionan un espacio de enseñanza y aprendizaje universitario en donde la reflexión individual, la colaboración y la atención personalizada se vuelven reales.

\section{Desarrollo: una experiencia innovadora en la enseñanza de "Periodismo en la Red"}

\subsection{El contexto innovador}

"Periodismo en la Red" es una asignatura que se presta a la innovación por su pretensión formativa dentro del Grado de Periodismo, con gran incidencia en el ejercicio profesional ya que los estudiantes desarrollan en el marco formativo universitario el trabajo que los comunicadores llevan a cabo a diario en las salas de redacción de los medios. La ventaja del periodismo en la red es el obligado uso de los ordenadores y como en toda producción noticiosa es indispensable el trabajo en equipo. Así, la información escrita por el profesional como parte de la construcción de un cuerpo de noticias, se hace colectiva y, es sobre esa base que el docente universitario debe actuar.

La Universidad Carlos III de Madrid y la empresa Adalid desarrollaron una plataforma de enseñanza-aprendizaje ad-hoc para esta asignatura. La plataforma de "Periodismo en la Red" se denomina "diariodepracticasuc3m.com" y permite generar diarios digitales en condiciones similares a las de los grandes diarios. Desde la citada plataforma se pueden crear textos, adjuntar imágenes, audios y videos, en fin, producir un periódico digital con todas las características que el mercado propone y exige.

La plataforma presenta dos caras. La trastienda, que es donde se produce el trabajo de diseño y de redacción y otra, la de lectura o de publicación que es visible para el lector. En la primera, con una estructura parecida al blog, el estudiante se encuentra con sus compañeros y es allí donde se interactúa a través de ordenadores para trabajar el diseño de la portada y las secciones. Se distribuyen los roles, se selecciona a los editores y se asumen responsabilidades como editores de cada una de las secciones o del periódico en general. Por supuesto toda esta labor de diseño y redacción implica el compromiso de comprender que lo que se escriba tiene que estar suficientemente contrastado. Lo interesante es observar que el periódico no tiene para el estudiante 
las mismas características que para el lector, que únicamente acude a la plataforma para su lectura.

Para efectos del estudio no solo se ha potenciado la plataforma, sino la propia dinámica de la creación de los periódicos, desde dos metodologías fundamentales: el portafolio y el CSCL, destacando en ambas el compromiso tutorial del docente.

\subsection{Portafolio y Aprendizaje Colaborativo Asistido por Ordenador (CSCL) como metodologías innovadoras}

El portfolio llamado en inglés portfolio assesment, portfolio process o teaching portfolio se fundamenta en la técnica americana denominada performance assessment, entendida como un enfoque de evaluación basado en el monitoreo progresivo de los avances del estudiante con relación a los resultados de aprendizaje en donde el alumno demuestra sus conocimientos o habilidades. En el caso de la enseñanza del periodismo, muestra los avances en dos frentes: los manejos de los estilos propios de la información periodística y por la otra, de la capacidad de investigación y uso de fuentes informativas.

Zhi-Feng (2007) propone la integración del aprendizaje personal del portfolio y el aprendizaje en grupo. Esta integración se compone de dos tipos de usuarios: el profesor, quien asigna las tareas y divide a los estudiantes en grupos, y los alumnos, a quienes se les pide que se familiaricen con los miembros de su grupo, para colaborar y completar los proyectos, y evaluar así los trabajos de otros equipos. A su vez, la fusión exige dos tipos de tareas: trabajos personales que se completan de forma individual, y las tareas de grupo, que serán terminadas por el equipo que ha sido conformado mediante la función de grupo colaborativo.

La reflexión juega un papel predominante en esta metodología. Según esta concepción, las competencias que se desarrollan van mucho más allá de las exigencias académicas ya que el profesional reflexivo es capaz de trascender la situación formativa al ámbito laboral. El portafolio se configura así como un registro de la reflexión estudiantil donde quedan guardadas las percepciones, problemas e interrogantes (Colén, Giné e Imbernón, 2006).

El CSCL implica un proceso formativo que sucede en pequeños grupos en interacción con un ordenador (Strijbos, Martens y Jochems, 2004). Como ejercicio socialintelectual el aprendizaje colaborativo pretende crear conocimiento a partir de la solución de un problema o tarea desde la interacción social. El grupo contesta a las preguntas, genera soluciones y cada miembro aprende de la experiencia. El principal objetivo es la construcción del conocimiento al generar a través de interacciones creativas la respuesta a la situación de estudio (Olivares, 2008).

Son dos los componentes para un aprendizaje colaborativo efectivo: motivación e interacción social. De esa manera, en la medida en que las actividades se asocien con necesidades e intereses de los estudiantes, la nueva producción se relacionará con conocimientos previos y así, se pasará de una "actividad-académica-colaborativa" a una "actividad académica-significativa-colaborativa".

Tanto el portafolio como el CSCL requieren un compromiso tutorial exigente por parte del docente. Ambas metodologías implican un seguimiento y supervisión cons- 
tante a los estudiantes, de manera personalizada o en pequeños grupos, les permitan avanzar en los aprendizajes y alcanzar los logros pretendidos. Así, en el estudio de Romero, Zurita Ortega y Zurita Molina (2010), en el que participan 290 estudiantes de Magisterio y Fisioterapia, los estudiantes otorgan un valor importante al uso del portafolio y de la tutoría para la experiencia del crédito ECTS, considerando que la tutoría debe ayudar ante las dificultades en el desarrollo de las asignaturas, facilitándoles focalizar su atención en aspectos de interés para los logros académicos y personales, valorando altamente que la tutoría esté integrada en la acción docente y en el itinerario formativo.

\section{Metodología}

El método que se ha aplicado en la investigación ha sido un estudio descriptivo de casos porque se ha descrito una situación educativa en un grupo de personas en un determinado momento (Hernández, Fernández y Baptista, 2010).

La población de la asignatura estuvo conformada por 66 estudiantes que asistían simultáneamente a la parte teórica y, luego, para efectos de la dinámica en el aula de informática, se subdividieron en 3 grupos de 22 alumnos. Uno de los grupos de 22 alumnos es nuestra muestra. Se conformaron cuatro subgrupos que tenían la responsabilidad de crear un periódico. Dos de los grupos con 5 alumnos y los otros dos con 6 estudiantes. Cuatro estudiantes asumieron la responsabilidad de ser editor del medio de comunicación y los demás estudiantes (18) tenían la responsabilidad de dirigir una sección de cada periódico.

Cada una de las clases prácticas (12 en total) conformada por dos partes fundamentales. Por una parte un acompañamiento y tutoría a cada grupo y, por la otra un acompañamiento y tutoría a cada estudiante. En cada sesión, el estudiante debía procesar al menos una información que sirviera para su sección u otra del periódico, pero todos debían colaborar con cada una de las secciones del periódico como condición sine qua non.

La labor del profesor se amparó en el portafolio y el CSCL, como estrategias docentes asumiendo el reto de hacer "Periodismo en la Red".

Durante las tres primeras semanas de las 14 que tiene el curso no se permitió a los alumnos publicar ningún trabajo, sino que, a pesar de estar esbozado el trabajo en la web y de hecho existían los medios con sus cabeceras y alguna fotografía y texto, se les contuvo de publicar para que viviesen la densidad del trabajo periodístico de crear y producir información relevante, suficientemente contrastada y bien referenciada. De esta manera podrían experimentar el sentir ético del periodismo y la responsabilidad con el texto presentado al lector. Si bien es cierto que, en el caso de esta experiencia de formación, son ellos mismos los lectores, por una parte, pero redactores con toda la presión y carga de responsabilidad por la otra.

Para llevar el registro de los datos se utilizó un baremo y una tabla que contenía las tutorías y notas de campo; para el aprendizaje colaborativo, baremo de actividades y de evaluación entre pares; para la evaluación del portafolio, un baremo de evaluación sobre la base de cinco indicadores: gramática, ortografía, pertinencia periodística, coherencia con los propósitos del medio de comunicación y manejo de la actualidad noticiosa. 
Todo ese registro de los portfolios era compartido sin la calificación con los alumnos para que ellos mismos tomaran decisiones de publicación, llevando de esta forma un elemento fáctico para sus discusiones de aprendizaje colaborativo y, en tutorías proceder a mejorar aquellos aspectos que fuera necesario resolver.

\section{Resultados}

El ejercitarse en situaciones que emulan a los propios medios de comunicación en la red, con sus ventajas y dificultades, la posibilidad de interactuar desde y con los ordenadores, el reto y la motivación que significa el crear un medio de comunicación desde el espacio en blanco y con las herramientas propias de la contemporaneidad son parte de este ejercicio de aprendizaje.

La experiencia innovadora en "Periodismo en la Red" se llevó a cabo durante el segundo semestre del curso 2011-2012, con una programación de cuatro horas semanales: dos teóricas y dos prácticas. Las sesiones prácticas se desarrollaron en la sala de ordenadores y en ellas se produjeron las tutorías, la revisión de los portafolios, la entrega de las correcciones de los avances para la publicación. Igualmente el profesor fue diseñando con los alumnos los parámetros fundamentales de los periódicos y se sentaron las bases para el flujo de trabajo. La Tabla 1 resume la dinámica de la clase.

Tabla 1. Síntesis de la experiencia innovadora

\begin{tabular}{|l|l|}
\hline Participantes: & 22 \\
\hline Grupos: & 4 grupos (dos de 5 estudiantes y dos de 6 estudiantes) \\
\hline \multicolumn{2}{|c|}{ Seguimiento y orientación docente } \\
\hline Sesiones de clase & 12 \\
\hline Número de avances para los periódicos & 170 \\
\hline Número de tutorías & 204 (17 por sesión de clase) \\
\hline \multicolumn{2}{|c|}{ Resultados: evidencias aprendizaje } \\
\hline Número de portafolios & 22 (uno por estudiante) \\
\hline Número de periódicos & 4 (uno por cada grupo) \\
\hline
\end{tabular}

La labor tutorial resolvió las dificultades de los estudiantes a la hora de producir información, especialmente sobre el propio medio de comunicación de cada grupo. La tutoría para los jefes de sección (todos los alumnos) y los editores (4 alumnos), se hizo en el propio salón de ordenadores, apartado de los demás compañeros. La tutoría fue útil para evaluar su desempeño y consultar sobre posibles soluciones periodísticas.

De la muestra intencional que se tomó, se produjeron cuatro periódicos en la red, cada uno con características diferentes fruto de la diversidad de intereses personales y estilos periodísticos de los estudiantes de cada grupo. Así, el grupo que originalmente se llamó " 16 " centró su interés por la estética, asumiendo que el color quedaría como acento y no como protagonista. Por ello, la selección de fotografías fue hecha en blanco y negro, razón por la cual, quien asumió el rol de editor cuidó sobre manera esos valores estéticos. Durante el proceso tutorial y de acompañamiento se habló muchas veces sobre los intereses del grupo. El periódico resultante tuvo como característica principal, ser un medio de comunicación fundamentalmente cultural, que lo 
convirtieron en un medio virtual con gran potencialidad, sobre todo por la comprensión que tuvo el grupo como espacio de aprendizaje en sí mismo desde un fluido manejo de la cooperación para el desarrollo de su trabajo.

Otro de los grupos, el "17", destacó por el marcado interés por el deporte, que destacó sobre las demás fuentes informativas, razón por la cual, la información no deportiva estuvo a cargo fundamentalmente de uno de los integrantes que, preocupado por la verosimilitud de sus noticias, consultó constantemente la opinión del profesor. Así, la tutoría no se oriento únicamente a la perspectiva formal de la enseñanza del periodismo, sino desde el punto de vista del fondo de las informaciones que se trataban.

El grupo "18" contaba con una estudiante Erasmus. El grupo decidió, dada la potencialidad de la estudiante, que se encargara del manejo de la sección internacional. Buena parte del interés del grupo se centró en buscar los elementos que fueran para el mercado internacional porque los demás integrantes tenían plena conciencia del rol de España y en especial de las islas Canarias en el turismo europeo y como tal sus esfuerzos se volcaron a crear un medio que destacara el turismo y sus posibilidades como factor informativo.

Finalmente, el grupo "19" asumió intereses más amplios en el ámbito de la información: generalistas, deportivas, culturales, políticas y económicas eran las fuentes que desarrolló este grupo con una destacada inclinación pormenorizada de cada una de sus integrantes en hacer ese tipo de información. De hecho, las tutorías con este grupo debieron centrarse en orientar que el trabajo de cada estudiante no se quedara en un tipo de fuente, sino que circulara su actividad por todas las fuentes y géneros periodísticos.

La labor docente se convirtió en asesoría permanente para la elaboración de los periódicos y, como tal, en lo que se podría llamar un trabajo editorial con una fuerte carga de enseñanza que trajo como consecuencia un manejo adecuado del ritmo de la clase en la que los estudiantes fueron los protagonistas de su aprendizaje.

La experiencia innovadora llevada a cabo en "Periodismo en la Red" ha puesto en conexión diferentes elementos. Por consiguiente, plataforma de teleformación, motivación al logro de los resultados, portafolio, aprendizaje colaborativo, acción tutorial y supervisión de trabajos periodísticos han sido los elementos fundamentales de la misma.

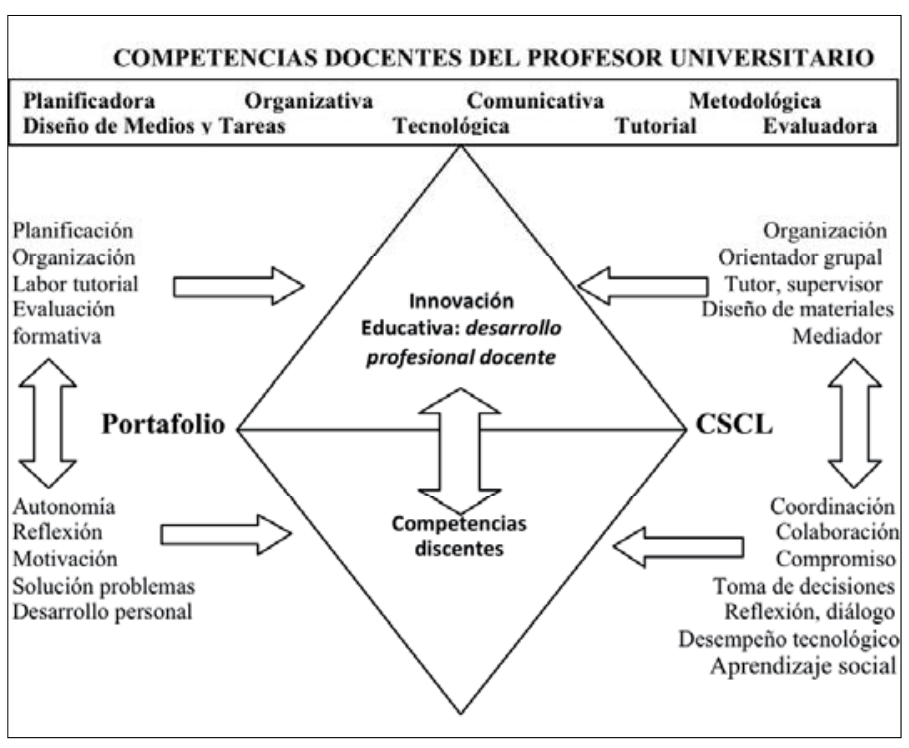

Figura 1: Modelo de innovación de la docencia universitaria 
Además de los resultados propios de la experiencia ya indicados, quisiéramos destacar como efecto de la innovación llevada a cabo el siguiente modelo que puede convertirse en lo sucesivo en un referente para la acción (Figura 1)

\section{Conclusiones}

Una de las conclusiones más interesante de la experiencia ha sido la puesta en común de los periódicos digitales. Ya no se trata de hacer prácticas por el mero hecho de escribir, si no de poner en colectivo los esfuerzos por destacar, ser originales en el tratamiento de la información, dar cabida a una realidad de que puede ser vista por millones de personas porque estará en la red. No caben las prácticas de corte y pega. Lo importante es ser periodista, tener a la ética de la profesión por delante, proponer informaciones para una sección de un periódico, pensar en la portada. Se trata de escribir para que el texto de la noticia sea valioso y pertinente.

Toda enseñanza requiere de una estructura consolidada, de oportunidades y condiciones sistemáticas que promuevan y potencien el aprendizaje de los estudiantes. Las metodologías centradas en el alumno, donde la responsabilidad recae en el aprendiz, han sido estudiadas con rigor científico. Los resultados avalan sus beneficios en el desarrollo de habilidades cognitivas, sociales y emocionales debido a que generan un aprendizaje profundo, significativo y duradero, facilitando así la transferencia a otros contextos.

El presente trabajo es el resultado de una primera aproximación al trabajo de metodologías activas en un aula universitaria con futuros profesionales del periodismo. El alcance del estudio se ha limitado a presentar un primer avance de la experiencia, que nos ha permitido generar un modelo de innovación con la finalidad de seguir profundizando en esta línea de investigación. Desde luego, nuestra experiencia presenta ciertas debilidades como son la no generalización de los resultados obtenidos y la no aplicación de instrumentos validados. Entre sus fortalezas, cabe resaltar el empleo del portafolio y el CSCL como metodologías que responden a los objetivos de la asignatura y a la vez, desarrollan competencias académicas y profesionales en los estudiantes, así como habilidades sociales y un sentido crítico y reflexivo del quehacer periodístico. Ambas metodologías acompañadas con una tutoría personalizada han favorecido el aprendizaje autónomo y continuo, la motivación y el interés, el rendimiento y el sentido crítico y reflexivo del conjunto de los estudiantes. Pero los beneficios de esta experiencia innovadora no son únicamente para el estudiante. El docente, sabedor de su compromiso con la profesión, puede encontrar en la innovación de su práctica un lugar de disfrute y desarrollo profesional.

\section{Referencias bibliográficas}

BUDAPEST-VIENNA DECLARATION (2010): "Budapest-Vienna on the European Higher Education Area". Disponible en: http://www.ond.vlaanderen.be/hogeronderwijs/bologna/2010_conference/documents/Budapest-Vienna_Declaration.pdf [Con acceso: 3 de noviembre de 2012]

COLÉN, María; GINÉ, Nuria; e IMBERNÓN, Francisco (2006): La carpeta de aprendizaje del alumnado universitario. Barcelona, Octaedro-ICE. 
HERNÁNDEZ, Roberto; FERNÁNDEZ, Carlos; y BAPTISTA, Pilar (2010): Metodología de la Investigación ( $5^{\text {a }}$ edición). México D.F., McGraw Hill.

IBÁÑEZ-MARTÍN, José Antonio (1990): "Dimensiones de la competencia profesional del profesor de universidad". Revista Española de Pedagogía, 186, pp. 239-257.

LÓPEZ, Ernesto (2012): "La tutoría en la universidad: una experiencia innovadora en el Grado de Educación Primaria". Revista de Docencia Universitaria, en prensa.

MEDINA, Antonio (coord., 2009): Innovación de la educación y de la docencia. Madrid, Ramón Areces-UNED.

OLIVARES, Orlando (2008): "Collaborative vs Cooperative Learning: The Instructor's Role in Computer Supported Collaborative Learning". En: ORVIS, K. \& LASSITER, A. (coords.): Computer-supported collaborative learning: Best practices and principles for instructors (pp. 20-39). New York, Information Science Publishing

RÖMER, Max y CAMILLI, Celia (2012): "El comentario periodístico. Una mirada teórica y didáctica desde la enseñanza universitaria". Revista de Comunicación Vivat Academia, 117, Año XIV, pp. 731-738.

ROMERO, Cipriano; ZURITA, Félix; y ZURITA, Félix (2010): "La autonomía y orientación en el EEES mediante el portafolio y la tutoría". Estudios Sobre Educación, 19, pp. 261-282.

STRIJBOS, Jan Willem; MARTENS, Rob; \& JOCHEMS, Wim (2004): “Designing for Interaction: Six Steps to Designing ComputerSupported Group-Based Learning". Computers \& Education, 42, pp. 403-424.

ZHI-FENG LIU, Eric (2007): "Developing a personal and group-based learning portfolio system". British Journal of Educational Technology, 38(6), pp. 1117-1121.

\section{Dr. Max RÖMER PIERETTI}

Universidad Carlos III Madrid

Departamento de Periodismo

Profesor asociado. Consultor de comunicaciones estratégicas y políticas a empresas, instituciones y políticos desde 1991

mroemer@hum.uc3m.es

\section{Celia CAMILLI TRUJILLO}

Centro Universitario Villanueva (adscrito a la Universidad Complutense de Madrid)

Profesora del Departamento de Pedagogía y Contextos Educativos

ccamilli@villanueva.edu

\section{Ernesto LÓPEZ GÓMEZ}

Centro Universitario Villanueva (adscrito a la Universidad Complutense de Madrid) Profesor del Departamento de Pedagogía y Contextos Educativos elopez@villanueva.edu 